\title{
Estimates of calf starter energy affected by consumption of nutrients. 1. Evaluation of models to predict changing digestion on energy content in calf starters
}

\author{
J. D. Quigley, ${ }^{*}$ W. Hu, J. R. Knapp, T. S. Dennis, F. X. Suarez-Mena, and T. M. Hill \\ Nurture Research Center, Provimi North America, Cargill Animal Nutrition, Brookville, OH 45309
}

\section{ABSTRACT}

Apparent total-tract digestibility data from 3 published studies with calves from 0 to 4 mo of age were used to evaluate National Research Council (2001) estimates of metabolizable energy (ME) in calf starters (CS). Calves $(n=83)$ or pens of calves $(n=24)$ were used in model development. In each study, 48 Holstein bull calves ( 2 to $3 \mathrm{~d}$ of age at initiation of each study) were fed varying amounts of milk replacer with CS and water for ad libitum consumption. Calf starters varied in nutrient content and form (pelleted, texturized, or mixed with $5 \%$ grass hay and fed as a total mixed ration). Apparent total-tract digestibility was measured at various ages from 3 to 16 wk. Feed and feces were collected from 20 calves per trial during 5 -d collection periods during the first $56 \mathrm{~d}$ of each trial. In 2 studies, calves were grouped in pens (4 calves/pen) for a second 56-d measurement period. Fecal collections were repeated occasionally during the second period. Totaltract digestibilities $(n=207)$ of neutral detergent fiber, nonfiber carbohydrates, crude protein, and fat were used to calculate digestible energy (DE) and ME in CS using equations from the 2001 Dairy National Research Council. Three modeling approaches were constructed to evaluate changing digestion of nutrients, DE, and ME in CS, including linear mixed models, broken-line regression, and exponential models. Linear mixed models provided best model fit statistics for digestion of crude protein, ether extract, neutral detergent fiber, and ME. Exponential models were optimal for digestion of dry matter and nonfiber carbohydrates. Linear mixed models were selected for evaluation of effects of intake on changing nutrient digestion from CS and amount of DE and ME available at various ages.

Key words: calf, digestion, energy, modeling

Received July 10, 2018.

Accepted November 27, 2018.

*Corresponding author. Jquigley@provimi-na.com

\section{INTRODUCTION}

Young dairy calves undergo fundamental changes in the source of nutrients in the first months of life. Maturation of the gastrointestinal tract, including development of fermentative capacity of the reticulorumen, occurs as calves transition from a diet consisting solely of highly digestible colostrum and milk to a postweaning diet consisting primarily of cereal grains and forages (Baldwin et al., 2004; Blum, 2006; Guilloteau et al., 2009). Production of VFA resulting from fermentation of carbohydrates early in life initiates rumen development, which accelerates the time at which calves are prepared for weaning (Warner et al., 1956; Sander et al., 1959).

Changing nutrient digestion with advancing age or intake of dry feed can be modeled using several techniques, including linear mixed models, broken-line regression, or nonlinear exponential models. The objective of this study was to evaluate 3 methods of modeling energy values of dry feeds (NRC, 2001) fed to young calves during the first 4 mo of life utilizing estimates of total-tract nutrient digestion.

\section{MATERIALS AND METHODS}

\section{Calves and Management}

Apparent total-tract digestibility (TTD) of nutrients was measured during 3 experiments (Table 1) published previously (Hill et al., 2016; Dennis et al., 2018; Quigley et al., 2018) and summarized in Quigley et al. (2019). Briefly, 48 newborn Holstein bull calves 2 to $3 \mathrm{~d}$ of age were used in trials for 56 (trial 1) or $112 \mathrm{~d}$ (trials 2 and 3). Experimental treatments and animal management are described elsewhere (Hill et al., 2016; Dennis et al., 2018; Quigley et al., 2018). In each experiment, a subset of 5 calves/treatment (d 0 to 56) or all pens (d 57 to 112) were used for digestibility measurements. Digestibility of calf starter DM (dDMcs), CP (dCPcs), ether extract (dEEcs), NDF (dNDFcs), and NFC (dNFCcs) were calculated from 
intake and fecal concentrations of chromic oxide (d 0 to 56 ) or acid insoluble ash (d 57 to 112) as described by Quigley et al. (2019).

Daily intakes of milk replacer (MR) and calf starter (CS) were measured by calf or pen. Average daily dry feed intake during the digestion period and cumulative dry feed intake from the beginning of the trial through the end of the digestion period were calculated. Description of experiments, experimental units, and diet information are in Quigley et al. (2019). Calculations of digestible energy in CS (DEcs), ME in CS (MEcs), and ratio of MEcs to ME estimated using NRC (2001) equations (MEratio) are also described in Quigley et al. (2019).

\section{Statistical Models}

Pearson correlation coefficients (Table 1) were generated to determine the relationship between dDMcs, dCPcs, dEEcs, dNDFcs, dNFCes, DEcs, MEcs, MEratio, and daily as well as cumulative CS intake of DM, $\mathrm{CP}$, ether extract, NDF, and NFC. Natural logarithm of daily and cumulative intake for each nutrient were also included. Effects of daily and cumulative nutrient intake on CS nutrient digestibility, DEcs, MEcs, and MEratio were evaluated using linear mixed models, broken-line regression, and exponential models.

Linear mixed models were evaluated as described by St-Pierre (2001). We evaluated multiple models:

$$
\begin{gathered}
\mathrm{Y}=\mathrm{I}+\text { form }+\mathrm{I} \times \text { form; } \\
\mathrm{Y}=\mathrm{I}+\text { form }+ \text { age }+\mathrm{I} \times \text { form} ; \\
\mathrm{Y}=\mathrm{I}+\text { form }+\mathrm{MRI}+\mathrm{I} \times \text { form} ;
\end{gathered}
$$

where $\mathrm{Y}=$ TTD of nutrients, DEcs, MEcs, and MEratio; $\mathrm{I}=$ daily or cumulative intake of DM, CP, ether extract, NDF, or NFC from CS or the natural logarithm of each term; age $=$ age (weeks); MRI $=$ milk replacer intake $(\mathrm{kg} / \mathrm{d})$; and form = dietary form [texturized CS (TEX), pelleted CS (PEL), or TMR], included in models as a fixed effect. Trial was included in all models as a random effect. Models were reduced when random covariance or random slope were not significant (St-Pierre, 2001). Values in figures were adjusted as outlined in St-Pierre (2001). The MIXED procedure of SAS (version 8, SAS Institute Inc., Cary, NC) does not provide an coefficient of determination $\left(\mathbf{R}^{2}\right)$ statistic that can be used to compare models; therefore, amount of variation accounted for by the selected model was estimated by regressing observed versus predicted values and calculating the $R^{2}$ value.
An exponential function was also evaluated. The model was $\mathrm{Y}=\mathrm{I}+\mathrm{A} \times\{1-\exp [-(\mathrm{K} \times \operatorname{ccsNFCI})]\}$, where $\mathrm{Y}=$ dependent variable (TTD of each nutrient, DEcs, MEcs, or MEratio), I = intercept, A = maximum value, $\mathrm{K}=$ rate of change, and ccsNFCI = cumulative intake $(\mathrm{kg})$ of NFC from d 0 to the end of the digestibility measurement period. Proc NLMIXED of SAS was used. Starting values were estimated and adjusted if models failed to converge. Fixed effects models with or without intercept were evaluated. Separate models including random effects of $\mathrm{A}, \mathrm{K}$, or both, were evaluated also. The $\mathrm{R}^{2}$ and adjusted $\mathrm{R}^{2}$ (AdjRsq) of the nonlinear regression and prediction error $(\mathbf{P E})$ were calculated in NLMIXED using the equations

$$
\begin{gathered}
\mathrm{R}^{2}=(\text { CTOTSS }- \text { SSERR }) / \text { CTOTSS } \\
\text { AdjRsq }=1-\left[\left(1-\mathrm{R}^{2}\right) \times(\mathrm{n}-1) /(\mathrm{n}-\mathrm{p}-1)\right], \\
\mathrm{PE}=\sqrt{(\text { SSERR } / \mathrm{n})},
\end{gathered}
$$

where CTOTSS $=$ corrected total sum of squares; SSERR $=$ error sum of squares; $\mathrm{n}=$ number of observations; $\mathrm{p}=$ number of parameters.

Broken-line regression procedures using Proc NLMIXED of SAS as described by Robbins et al. (2006) were evaluated. Dependent variables included TTD of nutrients, DEcs, MEcs, and MEratio. Independent variable was the natural logarithm of cumulative calf starter NFC intake (lnccsNFCI). One- and two-slope models were evaluated. Random effects for first-segment asymptote were evaluated also for the 1-slope model. The AdjRsq and PE were calculated as for exponential models.

For exponential and broken-line models, the model with the lowest Akaike information criterion and Bayesian information criterion was used to compare with other methods. Regression of observed versus predicted values and residual plots were generated as in Shah and Murphy (2006).

\section{RESULTS}

Five-day TTD measurements $(\mathrm{n}=207)$ were used in the data set, including 147 observations from 83 calves from 0 to $56 \mathrm{~d}$ and 60 observations from group pens during d 57 to 112. Intake of CS and mean nutrient digestibilities are reported elsewhere (Quigley et al., 2019).

Apparent TTD increased from low levels (except CP) as calves aged and CS intake increased. Pearson correlation coefficients (Table 1 ) indicated that lnccsNFCI was more highly related to dDMcs, dNFCcs, DEcs, and 
QUIGLEY ET AL.

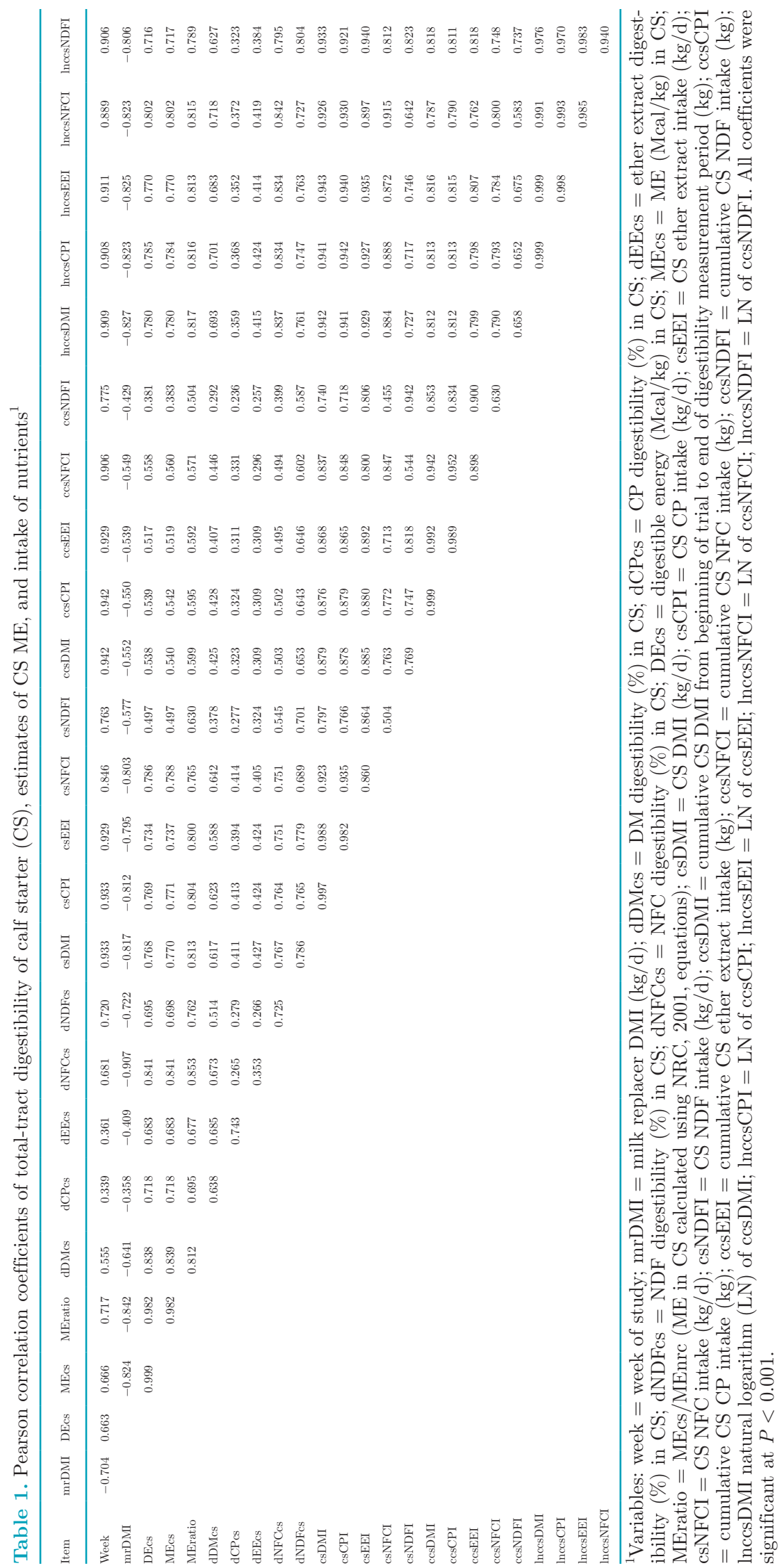


MEcs (Table 1) compared with age or daily intake of nutrients. Digestibility of CP was most highly correlated with average daily $\mathrm{CP}$ intake, whereas dEEcs and dNDFcs were most highly related to average daily DMI. The MEratio was most highly correlated with the natural logarithm of cumulative DMI. However, in all cases, lnccsNFCI was highly correlated with all variables, and therefore, lnccsNFCI was used for broken-line regression and linear mixed model, and cumulative NFC intake from CS (ccsNFCI) was used for the exponential model.

Fit statistics for linear mixed models, the best exponential, and the best broken-line regression models are in Table 2. Linear mixed model or exponential model resulted in best fit statistics for all nutrients, digestible energy, and ME estimates.
Parameter estimates for linear mixed models using lnccsNFCI and CS form are shown in Table 3. Generally, CS form or interaction of form $\times$ lnccsNFCI interactions were significant for TTD of nutrients, MEcs, and MEratio. Multiple exponential and brokenline regression models were evaluated and models with the lowest Akaike information criterion and Bayesian information criterion statistics and greatest adjusted $\mathrm{R}^{2}$ were selected, and parameter estimates are shown in Table 4. Of the exponential models, only dDMcs lacked an intercept. One slope broken-line models were selected for all independent variables except dNDFcs, DEcs, and MEcs, when the second slope was significant. Apparent TTD of DM, CP, ether extract, NDF, and NFC, DEcs, MEcs, and MEratio as well as model predictions are shown in Figure 1.

Table 2. Evaluation of linear mixed, exponential, and broken-line models of calf starter digestibility and estimates of calf starter digestible energy, ME, and MEratio

\begin{tabular}{|c|c|c|c|c|c|c|}
\hline \multirow[b]{2}{*}{ Variable $^{1} /$ model } & \multicolumn{4}{|c|}{ Fit statistics ${ }^{2}$} & \multicolumn{2}{|c|}{ 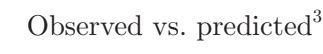 } \\
\hline & AIC & $\mathrm{BIC}$ & Adj. $R^{2}$ & $\mathrm{PE}$ & Slope & $\mathrm{R}^{2}$ \\
\hline \multicolumn{7}{|l|}{$\mathrm{dDMcs}$} \\
\hline Linear & -141.6 & -145.2 & 0.62 & 0.151 & 0.9538 & 0.638 \\
\hline Exponential & -281.0 & -271.3 & 0.51 & 0.175 & 0.9952 & 0.558 \\
\hline Broken-line & -151.0 & -137.7 & 0.57 & 0.165 & 0.9463 & 0.577 \\
\hline \multicolumn{7}{|l|}{$\mathrm{dCP} c s$} \\
\hline Linear & -1.3 & -3.1 & 0.24 & 0.217 & 0.9153 & 0.274 \\
\hline Exponential & -4.2 & 9.2 & 0.15 & 0.235 & 0.9028 & 0.162 \\
\hline Broken-line & 1.4 & 14.8 & 0.12 & 0.238 & 0.9001 & 0.135 \\
\hline \multicolumn{7}{|l|}{ dEEcs } \\
\hline Linear & 43.1 & 39.5 & 0.30 & 0.236 & 0.9050 & 0.337 \\
\hline Exponential & 40.2 & 53.6 & 0.18 & 0.262 & 0.8883 & 0.196 \\
\hline Broken-line & 43.7 & 57.0 & 0.17 & 0.264 & 0.8864 & 0.180 \\
\hline \multicolumn{7}{|l|}{ dNDFcs } \\
\hline Linear & -220.5 & -223.2 & 0.72 & 0.121 & 0.9238 & 0.734 \\
\hline Exponential & -187.1 & -175.0 & 0.51 & 0.164 & 0.9567 & 0.546 \\
\hline Broken-line & -162.8 & -146.2 & 0.53 & 0.159 & 0.8762 & 0.546 \\
\hline \multicolumn{7}{|l|}{ dNFCcs } \\
\hline Linear & -455.6 & -459.2 & 0.83 & 0.069 & 0.9934 & 0.842 \\
\hline Exponential & -506.9 & -494.8 & 0.79 & 0.078 & 1.0029 & 0.801 \\
\hline Broken-line & -482.7 & -469.4 & 0.82 & 0.074 & 0.9926 & 0.821 \\
\hline \multicolumn{7}{|l|}{ DEcs } \\
\hline Linear & 229.0 & 225.3 & 0.71 & 0.380 & 0.9867 & 0.721 \\
\hline Exponential & 220.4 & 233.7 & 0.68 & 0.404 & 0.9817 & 0.551 \\
\hline Broken-line & 230.9 & 247.6 & 0.67 & 0.413 & 0.9845 & 0.674 \\
\hline \multicolumn{7}{|l|}{ MEcs } \\
\hline Linear & 234.5 & 230.9 & 0.70 & 0.384 & 0.9822 & 0.715 \\
\hline Exponential & 219.9 & 233.3 & 0.68 & 0.404 & 0.9808 & 0.690 \\
\hline Broken-line & 234.4 & 251.1 & 0.66 & 0.416 & 0.9796 & 0.670 \\
\hline \multicolumn{7}{|l|}{ MEratio } \\
\hline Linear & -219.9 & -223.5 & 0.71 & 0.124 & 0.9816 & 0.721 \\
\hline Exponential & -258.8 & -245.4 & 0.71 & 0.127 & 0.9811 & 0.711 \\
\hline Broken-line & -242.6 & -229.3 & 0.68 & 0.132 & 0.9795 & 0.687 \\
\hline
\end{tabular}

${ }^{1}$ Dependent variable: $\mathrm{dDMcs}=\mathrm{DM}$ digestibility $(\%)$ in calf starter $(\mathrm{CS}) ; \mathrm{dCP} c \mathrm{C}=\mathrm{CP}$ digestibility $(\%)$ in $\mathrm{CS} ; \mathrm{dEEcs}=$ ether extract digestibility (\%) in CS; dNDFcs = NDF digestibility (\%) in CS; dNFCcs = NFC digestibility (\%) in CS; DEcs = digestible energy $(\mathrm{Mcal} / \mathrm{kg})$ in CS; MEcs = ME (Mcal $/ \mathrm{kg})$ in CS; MEratio = MEcs/MEnrc (ME in CS calculated using NRC, 2001, equations).

${ }^{2}$ Fit statistics: $\mathrm{AIC}=$ Akaike information criterion; $\mathrm{BIC}=$ Bayesian information criterion; $\mathrm{PE}=$ square root of (model sum of squares for error/number of observations); Adj. $\mathrm{R}^{2}=$ adjusted $\mathrm{R}^{2}$.

${ }^{3}$ Regression of observed versus predicted values. Slope $=$ slope of regression through the origin (intercept $=0$ ); $\mathrm{R}^{2}=\mathrm{R}^{2}$ of regression including intercept and slope. 


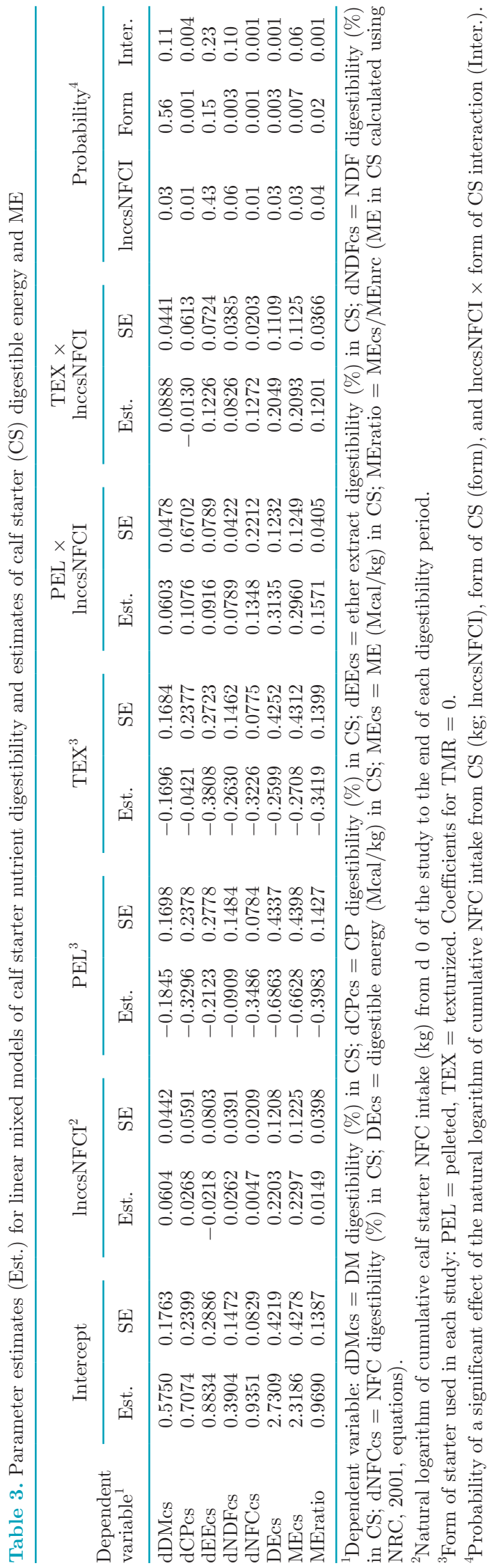

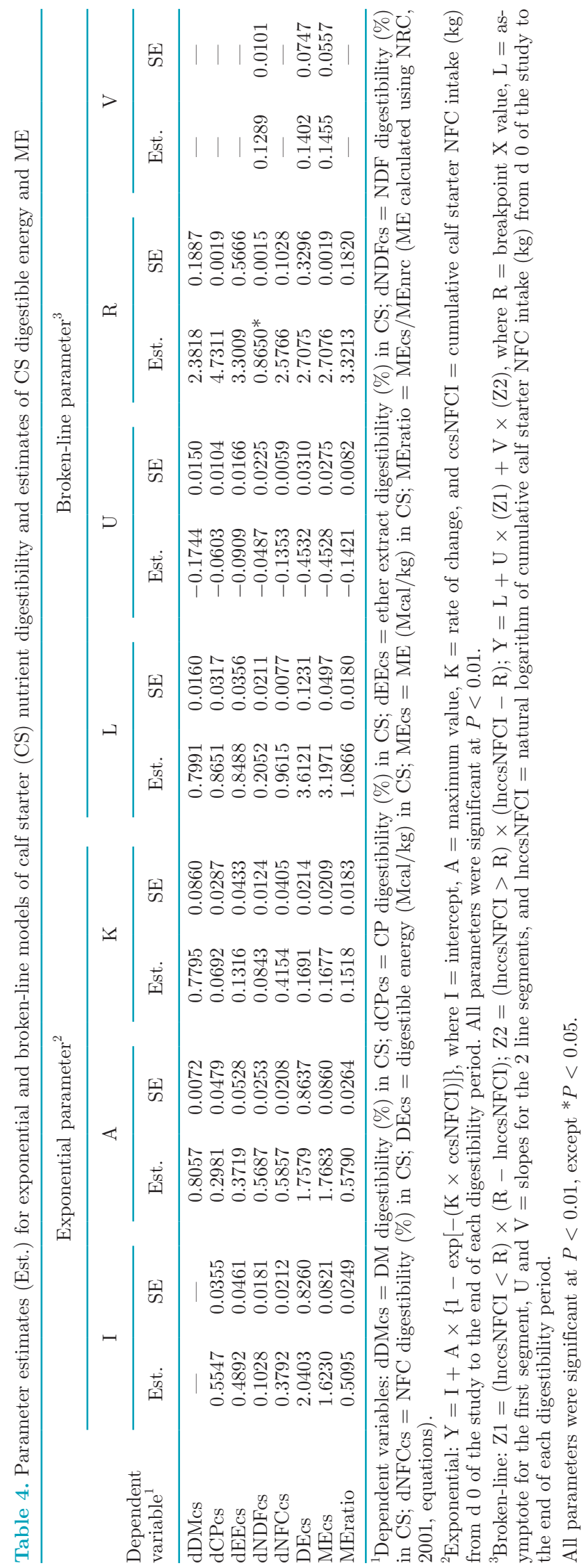


Exponential and broken-line regression models approached maximal (asymptote) for TTD of nutrients, MEcs, or MEratio at different intakes of cumulative NFC (Table 5). Broken-line regression of dNDFcs, DEcs, and MEcs included 2 slopes, indicating that these variables increased throughout the range of data.

Graphs of observed versus predicted (Figure 2) of MEcs for linear mixed models, exponential models, and broken-line models indicate that the linear mixed model generally resulted in the best prediction of MEcs. Similar plots for TTD of nutrients and MEratio supported the conclusion that the linear mixed model (using lnccSNFCI) provided the best model of the observed data (data not shown).

Calculated ME in TEX, using NRC (2001) equations, were $3.25,3.22$, and $3.26 \mathrm{Mcal} / \mathrm{kg}$ in the 3 trials comprising our data set. The PEL used as one treatment in trial 2 contained $2.92 \mathrm{Mcal} / \mathrm{kg}$. Addition of $5 \%$ hay during the grower period (d 57 to 112) reduced the amount of ME in all CS.

We calculated MEratio to estimate the point at which calculated ME (MEcs) was similar to the value estimated by NRC (MEnrc). For linear mixed, exponential, and broken-line models, MEratio equaled 1.0 $(\mathrm{MEcs}=\mathrm{MEnrc})$ at $15.8,12.4$, and $15.1 \mathrm{~kg}$ of ccsNFCI, respectively. Maximal MEratio was reached at 103, 108, and $108 \%$, respectively.

\section{DISCUSSION}

Digestion of nutrients (DM, CP, ether extract, NDF, and NFC) from CS was low early in life and increased with advancing age and intake. These observations are consistent with limited rumen development (Hibbs et al., 1956; Warner et al., 1956; Stobo et al., 1966), limited NDF (Dennis et al., 2018; Quigley et al., 2018),
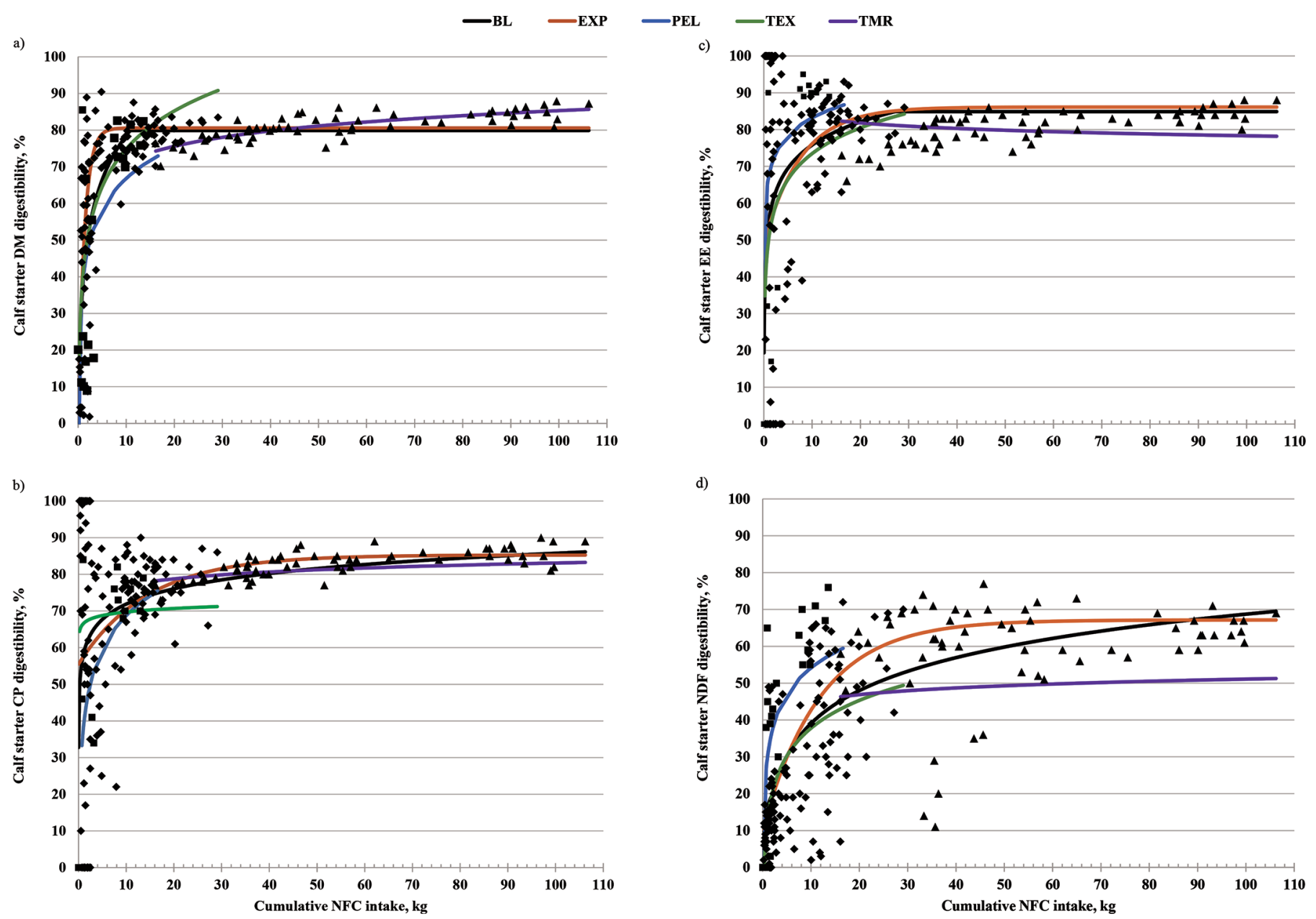

Figure 1. Broken-line (BL), exponential (EXP), or linear mixed model estimates of nutrient digestibility and calf starter ME in calves fed pelleted (PEL) or texturized (TEX) calf starters without or with (TMR) 5\% grass hay. Values are adjusted as described by St-Pierre (2001). (a-e) Dry matter, CP, ether extract (EE), NDF, and NFC digestibilities, respectively; (f) calculated calf starter digestible energy (DE); (g) calculated calf starter ME; and (h) ratio of calf starter ME to ME calculated using NRC (2001) equations (ME ratio). 
and starch digestion early in life (Morrill et al., 1970; Guilloteau et al., 1984). Intake of fermentable carbohydrate initiates rumen development (Warner et al., 1956; Sander et al., 1959), and increasing age and starch intake influence pancreatic development and intestinal starch digestion (Morrill et al., 1970; Guilloteau et al., 1984). Therefore, our observation that intake of NFC rather than age or intake of MR was a better predictor of changing digestion and estimation of MEcs is consistent with existing theories of digestive development in young calves. Calves fed $>1 \mathrm{~kg}$ of MR solids per day in these studies consumed less CS early in life and TTD of most nutrients from CS were lower, likely due to delayed rumen development.

Pearson correlation coefficients (Table 1) were used to evaluate potential independent variables. Natural logarithm of cumulative NFC intake was most highly correlated with TTD of DM and NFC and ME in CS, suggesting a period of digestive development followed by maturation of digestive capacity. Inclusion of additional independent variables such as natural logarithm of cumulative NDF intake, age, or MR intake did not significantly improve model fit, and therefore, a single independent variable was used. For linear mixed models and broken-line regression models, lnccsNFCI was used; for exponential models, ccsNFCI was used.

Numerous approaches are available to model nutrient requirements such as broken-line regression modeling of AA (Robbins et al., 2006; Pesti et al., 2009) or growth accretion models such as Gompertz curves (Tjørve and Tjørve, 2017) and exponential models. Development of apparent total-tract digestion of nutrients is likely multi-factorial and affected by influences such as rumen $\mathrm{pH}$, rate of passage, and feed particle size. However,
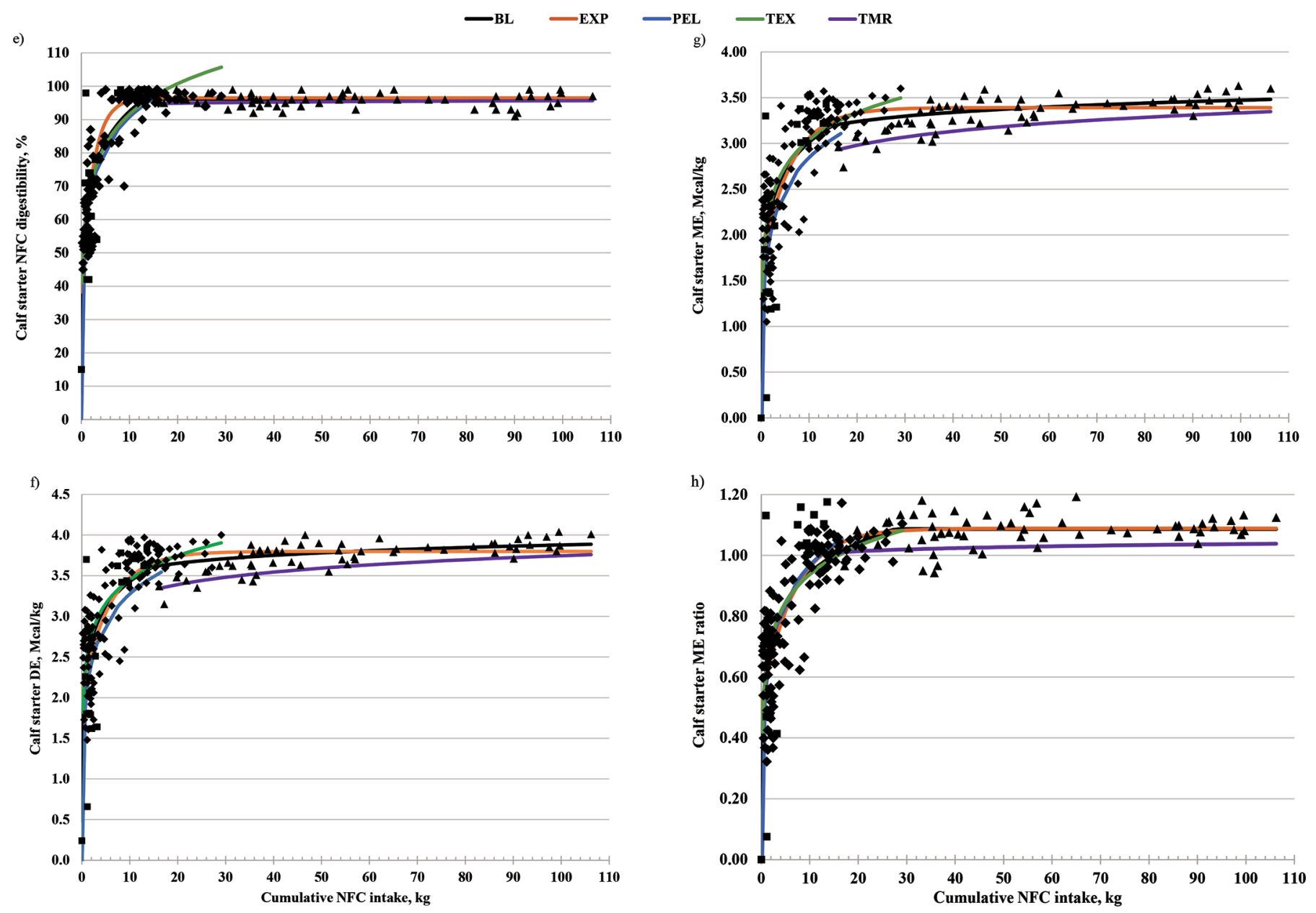

Figure 1 (Continued). Broken-line (BL), exponential (EXP), or linear mixed model estimates of nutrient digestibility and calf starter ME in calves fed pelleted (PEL) or texturized (TEX) calf starters without or with (TMR) $5 \%$ grass hay. Values are adjusted as described by St-Pierre (2001). (a-e) Dry matter, CP, ether extract (EE), NDF, and NFC digestibilities, respectively; (f) calculated calf starter digestible energy (DE); (g) calculated calf starter ME; and (h) ratio of calf starter ME to ME calculated using NRC (2001) equations (ME ratio). 
all selected models accounted for significant variation in changing digestion of most nutrients and estimated digestible energy and ME.

Form of ration was significant in most linear mixed models (except dDMcs and dEEcs), indicating that form of CS influenced TTD. This was primarily due to the addition of $5 \%$ hay to make TMR during d 57 to 112. The effect of TEX versus PEL was only significant for $\mathrm{dCP} c s$ and dNDFcs during d 0 to 56 ; other measures were unaffected by ration form during the first 2 mo of life. This also suggests that changing diets on d 56 from PEL or TEX to a TMR containing 5\% hay introduced variability into our data. Therefore, the ratio of MEcs to MEnrc (i.e., MEratio) may be a more appropriate indicator of degree of maturation of digestive capacity. The MEratio for linear mixed, exponential, and brokenline models reached $100 \%$ at $15.8,12.4$, and $15.1 \mathrm{~kg}$ of ccsNFCI, respectively. Maximal MEratio was 103, 108 , and 108\%, respectively. The MEratio predicted by TMR in the linear mixed model was lower than predictions by exponential and broken-line models and may have reflected the nature of changing diet during the grower phases of trials 2 and 3. The MEratio for TMR was essentially constant at $103 \%$, indicating that ME predicted by NRC (2001) was similar to the ME calculated from TTD measures.

Exponential and linear mixed models explained a similar amount of variation in digestibility data and estimates of calf starter ME as indicated by fit statistics in Table 2. Broken-line regression analyses were associated with greater error and lower $\mathrm{R}^{2}$ values. Linear mixed models included effects of ration form (PEL, TEX, or TMR). Neither exponential nor broken-line models contained indicator variables for ration form, as many models would not converge due to limited data set size.

Exponential and broken-line models with one slope reach a maximal value (asymptote), indicating maturation of digestive capacity. Table 5 contains calculated ccsNFCI at which no further increase in digestion occurred. Also reported is kilograms of ccsNFCI at which the incremental increase was $\leq 1 \%$ from the previous kilograms of ccsNFCI, indicating a minimal change in digestion. In some cases, estimates increased with increasing ccsNFCI beyond the range of our data, though biological significance was small. We chose a $1 \%$ increase to reflect that point at which minimal biological change occurred in digestion, recognizing that this selection was arbitrary. The ccsNFCI at maximal digestion varied by model and nutrient, and ranged from 12 to $130 \mathrm{~kg}$ of ccsNFCI. However, the point at which incremental increase in digestion or MEcs was $\leq 1 \%$ occurred when calves consumed 7 to $15 \mathrm{~kg}$ of ccsNFCI, except digestion of NDF, which increased $>1 \%$ per unit of ccsNFCI to $25 \mathrm{~kg}$.

Predicted versus observed and residual plots (Figure 2 ) indicated error around the prediction of MEcs in each model. Errors associated with predicting MEcs were the sum of errors in measuring DMI, errors associated with measurement and assumptions made in estimating chemical composition of feeds and feces, error associated with partitioning digestion between MR and CS before weaning, and estimating digestibility of nutrients using marker methods. We chose not to remove statistical outliers from our data, though some individual observations appeared to warrant removal.

Table 5. Estimates of intake of cumulative NFC $(\mathrm{kg})$ from calf starter at which maximal digestion or digestible energy and ME values were obtained using exponential or broken-line models

\begin{tabular}{|c|c|c|c|c|}
\hline \multirow{2}{*}{$\begin{array}{l}\text { Dependent } \\
\text { variable }^{1}\end{array}$} & \multicolumn{2}{|c|}{ Exponential } & \multicolumn{2}{|c|}{ Broken-line } \\
\hline & Maximal $^{2}$ & $\leq 1 \%$ increase $^{3}$ & Maximal & $\leq 1 \%$ increase \\
\hline dDMcs & 13 & 7 & 12 & 12 \\
\hline $\mathrm{dCP} c \mathrm{~s}$ & 130 & 15 & 114 & 9 \\
\hline dEEcs & 68 & 14 & 28 & 12 \\
\hline $\mathrm{dNDFCs}$ & 111 & 25 & $\mathrm{ND}^{4}$ & 25 \\
\hline dNFCes & 23 & 9 & 13 & 14 \\
\hline DEcs & 62 & 21 & 15 & 15 \\
\hline MEcs & 63 & 14 & 15 & 15 \\
\hline MEratio & 13 & 15 & 15 & 15 \\
\hline
\end{tabular}

${ }^{1}$ Dependent variable: $\mathrm{dDMcs}=\mathrm{DM}$ digestibility (\%) in calf starter $(\mathrm{CS})$; dCPcs = CP digestibility (\%) in $\mathrm{CS}$; dEEcs = ether extract digestibility (\%) in CS; dNDFcs = NDF digestibility (\%) in CS; dNFCcs = NFC digestibility (\%) in CS; DEcs = digestible energy $(\mathrm{Mcal} / \mathrm{kg})$ in CS; MEcs = ME $(\mathrm{Mcal} / \mathrm{kg})$ in CS; MEratio = MEcs/MEnrc (ME calculated using NRC, 2001, equations).

${ }^{2}$ Intake (kg) of cumulative calf starter NFC (ccsNFCI) at which no further increase in dependent variable occurred.

${ }^{3}$ The ccsNFCI at which there was $\leq 1 \%$ increase in value of the dependent value from previous kilograms of ccsNFCI.

${ }^{4} \mathrm{ND}=$ not determined for 2-slope model. 
a)

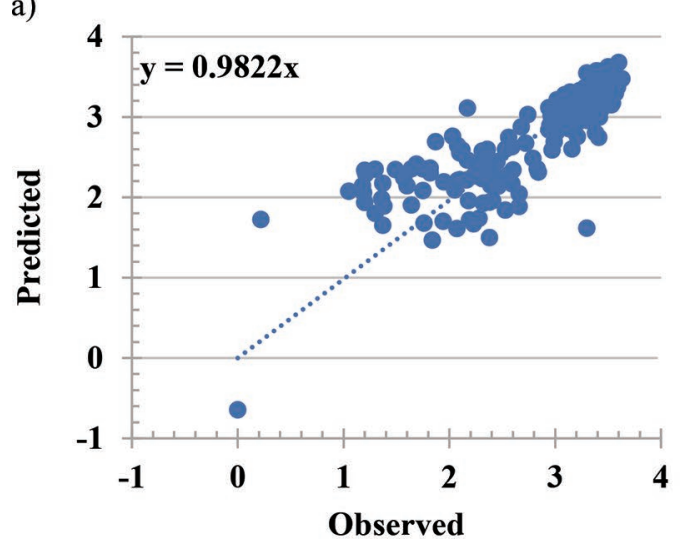

c)

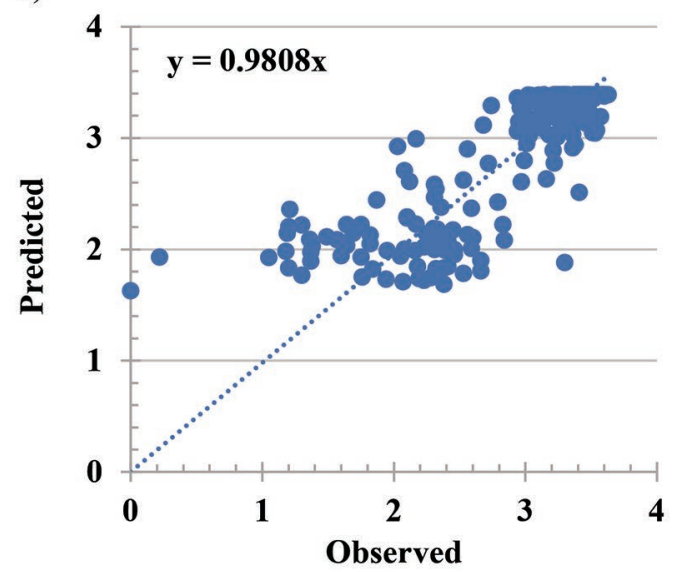

e)

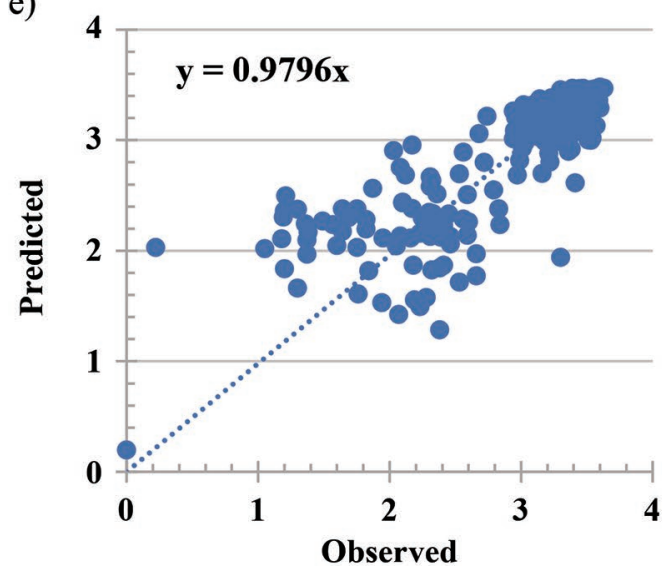

b)

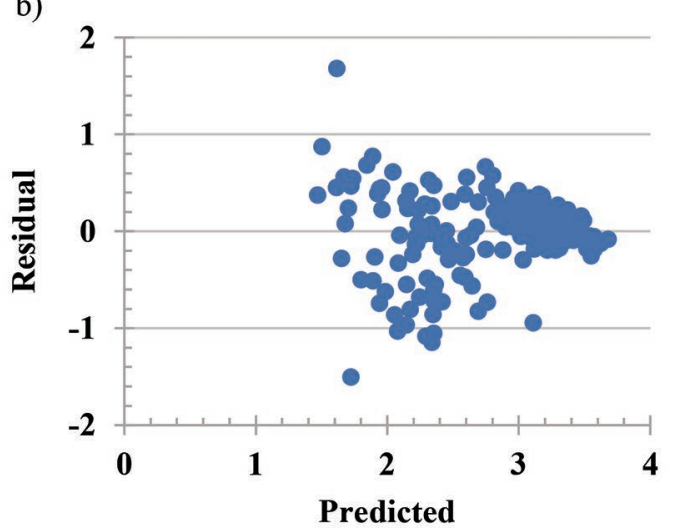

d)
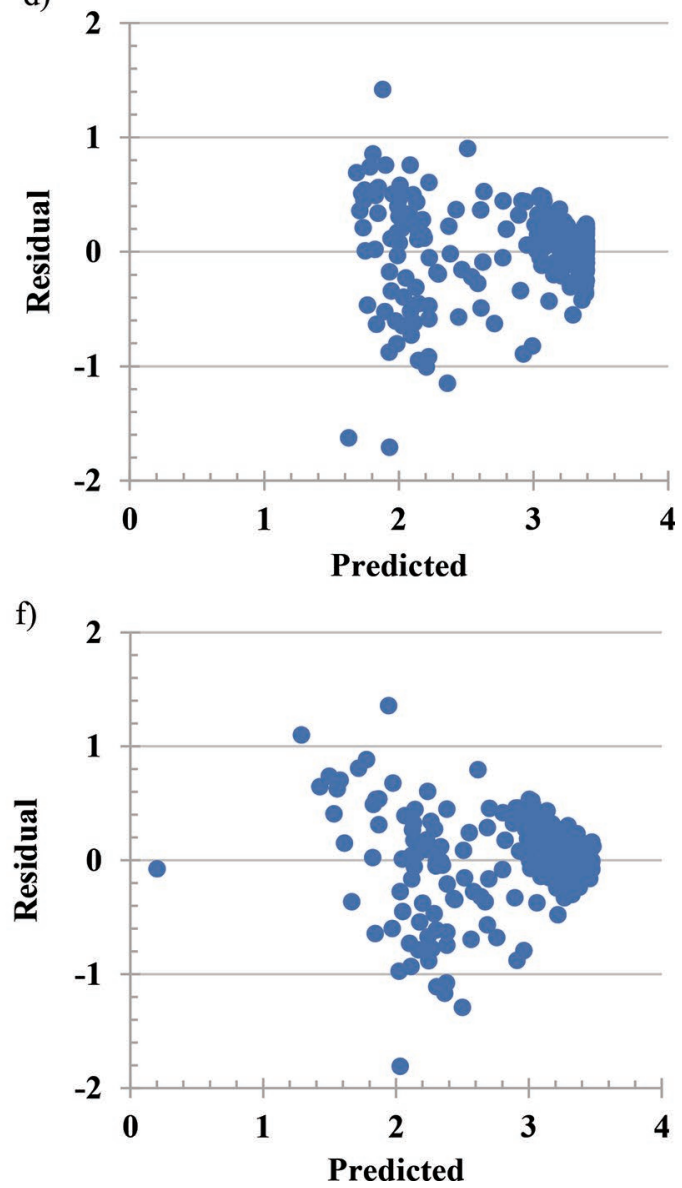

Figure 2. Predicted versus observed $\mathrm{ME}(\mathrm{Mcal} / \mathrm{kg})$ and residual versus predicted plots using linear mixed model, exponential, and brokenline models (a/b, c/d, e/f, respectively).

\section{CONCLUSIONS}

Changing digestion with increasing NFC was adequately modeled using linear mixed models with ration type and lnccsNFCI as independent variables. Exponential model of changing digestion also adequately described changing digestion with increasing intake.
Broken-line regression models were less descriptive. Linear mixed models were selected for further analysis.

\section{ACKNOWLEDGMENTS}

The authors gratefully acknowledge the input of the Farm Crew at the Nurture Research Center and assis- 
tance and advice of A. Nayeri and M. Baldin (Provimi) in design and data interpretation. The research was wholly funded by Provimi North America, a division of Cargill Animal Nutrition.

\section{REFERENCES}

Baldwin, R. L., K. R. McLeod, J. L. Klotz, and R. N. Heitmann. 2004. Rumen development, intestinal growth and hepatic metabolism in the pre- and postweaning ruminant. J. Dairy Sci. 87:(E. Suppl.):E55-E65.

Blum, J. W. 2006. Nutritional physiology of neonatal calves. J. Anim. Physiol. Anim. Nutr. (Berl.) 90:1-11.

Dennis, T. S., F. X. Suarez-Mena, T. M. Hill, J. D. Quigley, R. L. Schlotterbeck, and L. Hulbert. 2018. Effect of milk replacer feeding rate, age at weaning, and method of reducing milk replacer to weaning on digestion, performance, rumination, and activity in dairy calves to 4 months of age. J. Dairy Sci. 101:268-278.

Guilloteau, P., T. Corring, R. Toulec, and J. Robelin. 1984. Enzyme potentialities of the abomasum and pancreas of the calf. I. Effect of age in the preruminant. Reprod. Nutr. Dev. 24:315-325.

Guilloteau, P., R. Zabielski, and J. W. Blum. 2009. Gastrointestinal tract and digestion in the young ruminant: Ontogenesis, adaptations, consequences and manipulations. J. Physiol. Pharmacol. 60(Suppl. 3):37-46.

Hibbs, J. W., H. R. Conrad, W. D. Pounden, and N. Frank. 1956. A high roughage system for raising calves based on early development of rumen function. 6. Influence of hay to grain ratio on calf performance, rumen development, and certain blood changes. J. Dairy Sci. 39:171-179.

Hill, T. M., J. D. Quigley, F. X. Suarez-Mena, H. G. Bateman II, and R. L. Schlotterbeck. 2016. Effect of milk replacer feeding rate and functional fatty acids on dairy calf performance and digestion of nutrients. J. Dairy Sci. 99:6352-6361.

Morrill, J. L., W. E. Stewart, R. J. McCormick, and H. C. Fryer. 1970. Pancreatic amylase secretion by young calves. J. Dairy Sci. $53: 72-78$
NRC. 2001. Nutrient Requirements of Dairy Cattle. 7th rev. ed. Natl. Acad. Sci., Washington, DC.

Pesti, G. M., D. Vedenov, J. A. Cason, and L. Billard. 2009. A comparison of methods to estimate nutritional requirements from experimental data. Br. Poult. Sci. 50:16-32.

Quigley, J. D., T. M. Hill, T. S. Dennis, F. X. Suarez-Mena, and R. L. Schlotterbeck. 2018. Effects of feeding milk replacer at 2 rates with pelleted low-starch or texturized high-starch starters on calf performance and digestion. J. Dairy Sci. 101:5937-5948.

Quigley, J. D., T. M. Hill, W. Hu, T. S. Dennis, F. X. Suarez-Mena, and J. R. Knapp. 2019. Estimates of calf starter energy affected by consumption of nutrients. 2. Effect of changing digestion on energy in calf starters. J. Dairy Sci. 102:2242-2253. https://doi.org/10 .3168/jds.2018-15354.

Robbins, K. R., A. M. Saxton, and L. L. Southern. 2006. Estimation of nutrient requirements using broken-line regression analysis. J. Anim. Sci. 84(E. Suppl.):E155-E165.

Sander, E. G., R. G. Warner, H. N. Harrison, and J. K. Loosli. 1959. The stimulatory effect of sodium butyrate and sodium propionate on the development of rumen mucosa in the young calf. J. Dairy Sci. 42:1600-1605.

Shah, M. A., and M. R. Murphy. 2006. Development and evaluation of models to predict the feed intake of dairy cows in early lactation. J. Dairy Sci. 89:294-306.

St-Pierre, N. R. 2001. Invited Review: Integrating quantitative findings from multiple studies using mixed model methodology. J. Dairy Sci. 84:741-755.

Stobo, I. J. F., J. H. B. Roy, and H. J. Gaston. 1966. Rumen development in the calf. I. The effect of diets containing different proportions of concentrates to hay on rumen development. Br. J. Nutr. 20:171-188.

Tjørve, K. M. C., and E. Tjørve. 2017. The use of Gompertz models in growth analyses, and new Gompertz-model approach: An addition to the Unified-Richards family. PLoS One 12:e178691. https:/ doi.org/10.1371/journal.pone.0178691.

Warner, R. G., W. P. Flatt, and J. K. Loosli. 1956. Dietary factors influencing the development of the ruminant stomach. J. Agric. Food Chem. 4:788-792. 